\title{
Dickkopf-1 (Dkk-1) in plasma and synovial fluid is inversely correlated with radiographic severity of knee osteoarthritis patients
}

\author{
Sittisak Honsawek ${ }^{1 *}$, Aree Tanavalee ${ }^{2}$, Pongsak Yuktanandana², Srihatach Ngarmukos ${ }^{2}$, Natthaphon Saetan ${ }^{1}$, \\ Saran Tantavisut ${ }^{2}$
}

\begin{abstract}
Background: Osteoarthritis $(\mathrm{OA})$ is a common degenerative joint disease causing pain, stiffness, reduced motion, swelling, crepitus, and disability. Dickkopf-1 (Dkk-1) is a critical mediator of osteoblastogenesis and regulates the joint remodeling. The aim of this study was to examine plasma and synovial fluid Dkk-1 levels of patients with primary knee $O A$ and to investigate their relationship with disease severity.

Methods: Thirty-five patients aged 55-83 years with knee OA and 15 healthy individuals were recruited into this study. Disease severity was determined using weight-bearing anteroposterior radiographs of the affected knee. The radiological grading of OA in the knee was performed according to the Kellgren-Lawrence grading system. Dkk-1 levels in both plasma and synovial fluid were evaluated using enzyme-linked immunosorbent assay.

Results: The average concentration of circulating Dkk-1 in the knee OA patients was remarkably lower than that of healthy controls $(396.0 \pm 258.8,95 \% \mathrm{Cl} 307.1-484.9$ vs $2348.8 \pm 2051.5,95 \% \mathrm{Cl} 1164.3-3533.3 \mathrm{pg} / \mathrm{ml}, \mathrm{p}<0.0001)$. Dkk-1 levels in synovial fluid were significantly lower than in paired plasma samples $(58.6 \pm 31.8,95 \% \mathrm{Cl}$ 47.7-69.6 vs $396.0 \pm 258.8,95 \% \mathrm{Cl} 307.1-484.9 \mathrm{pg} / \mathrm{ml}, \mathrm{p}<0.001)$. Furthermore, both plasma and synovial fluid Dkk-1 levels were inversely correlated with radiographic severity $(r=-0.78, p<0.001$ and $r=-0.42, p=0.01$, respectively). Plasma Dkk-1 levels were also significantly correlated with synovial fluid Dkk-1 levels $(r=0.72, p<0.001)$.

Conclusions: Dkk-1 levels in plasma and synovial fluid are inversely related to the severity of joint damage in knee OA. Dkk-1 could serve as a biochemical marker for determining disease severity and might play a potential role in the pathogenesis of the degenerative process of OA.
\end{abstract}

\section{Background}

Osteoarthritis (OA) is the most prevalent joint disease causing pain, stiffness, reduced motion, swelling, crepitus, and disability. It is characterized by the progressive destruction of articular cartilage with joint-space narrowing, osteophyte formation, subchondral sclerosis, and synovitis [1]. The knee is the most clinically significant site of primary osteoarthritis involvement. One of the current methods to evaluate the affected joint is radiological assessment which reflects disease severity by grading the joint degeneration. The Kellgren-Lawrence

\footnotetext{
* Correspondence: sittisak.h@chula.ac.th

'Department of Biochemistry, Faculty of Medicine, Chulalongkorn University, Bangkok 10330, Thailand

Full list of author information is available at the end of the article
}

grading scale representing disease severity has been the most widely used system [2]. The etiology and pathogenesis of OA remain poorly understood, but have been associated with several physiological factors such as obesity and aging [3]. Nevertheless, biochemical factors have by now been recognized as playing an important role in OA development.

Secreted glycoproteins of the Wingless (Wnt) signaling pathway are crucial regulators of cell growth and survival in a variety of human cell types. Wnt ligands bind to a receptor complex encompassing a member of the Frizzled family of seven transmembrane proteins and the co-receptor, low-density lipoprotein (LDL) receptorrelated proteins (LRP5/6) [4]. In the canonical Wnt/ $\beta$-catenin signaling pathway, receptor activation results
C Biomed Central

(c) 2010 Honsawek et al; licensee BioMed Central Ltd. This is an Open Access article distributed under the terms of the Creative Commons Attribution License (http://creativecommons.org/licenses/by/2.0), which permits unrestricted use, distribution, and reproduction in any medium, provided the original work is properly cited. 
in a stabilization of $\beta$-catenin, which accumulates and translocates into the nucleus to activate target gene expression. Dickkopf-1 (Dkk-1) is a secreted protein that has been defined as a direct inhibitor of Wnt/ $\beta$-catenin signaling by interacting with the LRP $5 / 6$ coreceptors of frizzled [5,6]. Dkk-1 is a critical mediator of osteoblastogenesis and regulates the formation of the skeleton during the development of the embryo [7]. More recent studies have suggested a potential role of Dkk-1 in malignant bone disease and arthritis [8-10]. Uderhardt et al. have shown that inhibition of Dkk-1 effectively reduces bone erosion of sacroiliac joints [11]. In addition, Diarra and colleagues have documented that blockade of Dkk-1 reverses the bone-destructive pattern in a mouse model of rheumatoid arthritis to the bone-forming pattern of OA [10], indicating that Dkk-1 is a central regulator of joint remodeling. Recently, elevated circulating Dkk-1 levels have been associated with delayed progression of radiographic hip OA in women [12]. Furthermore, growing evidence has proposed an association between deregulated Wnt signaling components and joint disorders in OA cartilage chondrocyte cultures [13].

Even though circulating and/or synovial fluid levels of several cytokines have been investigated in patients with knee OA, there have not been any reports on the association of circulating and synovial fluid levels of Dkk-1 with disease activity in primary knee OA [14-18]. We have hypothesized that Dkk-1 in plasma and synovial fluid might be associated with the severity of clinical outcomes in knee OA patients. To prove this hypothesis, we have investigated the plasma and synovial fluid levels of Dkk-1 in knee OA patients and healthy controls. The aim of the present study was to evaluate, for the first time in the literature, both plasma and synovial fluid levels of Dkk-1 in patients with primary knee OA, and examine the possible relationships between plasma and synovial fluid Dkk-1 with the radiographic grading of knee osteoarthritis.

\section{Methods}

\section{Study participants}

This study was approved by the Institutional Review Board on Human Research of the Faculty of Medicine, Chulalongkorn University and was conducted in agreement with the Declaration of Helsinki. Written informed consent was obtained from the patients and healthy volunteers prior to their participation in this study.

Thirty-five patients aged 55 to 83 years with primary knee osteoarthritis (26 females and 9 males; mean age $68.8 \pm 8.2$ years) according to the criteria of the American College of Rheumatology were enrolled in the study. The severity of the disease was determined using weightbearing anteroposterior radiographs of the affected knee. Knee radiographs were evaluated according to the
Kellgren and Lawrence classification [2]: grade 1, doubtful narrowing of joint space and possible osteophytic lipping; grade 2, definite osteophytes and possible narrowing of joint space; grade 3, moderate multiple osteophytes, definite narrowing of joint space, some sclerosis and possible deformity of bone contour; grade 4, large osteophytes, marked narrowing of joint space, severe sclerosis and definite deformity of bone contour. The grading scale used for analysis was the one found higher upon comparison between both knees. We also recruited 15 gender and age matched subjects ( 10 females and 5 males; mean age $67.5 \pm 4.6$ years) with normal knee radiographs as controls. None of the participants had underlying diseases such as diabetes, histories of corticosteroid medication, other forms of arthritis, cancer, or other chronic inflammatory diseases.

\section{Laboratory methods}

Synovial fluid was aspirated from the affected knee using sterile knee puncture just prior to surgery, when a total knee replacement was performed, centrifuged to remove cells and joint debris and stored immediately at $-80^{\circ} \mathrm{C}$ until the day of measurement. No synovial fluid was extracted from the controls due to ethical concerns. Venous blood samples collected from the same patients on the day of surgery were centrifuged and stored at $-80^{\circ} \mathrm{C}$ until utilized. Double-blind quantitative detection of Dkk-1 in plasma and synovial fluid was performed by sandwich enzyme-linked immunosorbent assay (ELISA) using a commercially available test kit according to the manufacturer's protocol (Quantikine, R\&D Systems, Minneapolis, MN). Briefly, standards of recombinant human Dkk-1, plasma, and synovial fluid samples prediluted 1:4 in assay buffer were added to 96-well microtiter plates precoated with mouse monoclonal antibody against Dkk-1 and incubated for 2 hours at room temperature. The wells were then washed four times with washing buffer and incubated for 2 hours at room temperature with a horseradish peroxidase-conjugated goat polyclonal antibody against Dkk-1. After four washes, substrate solution was added to each well, and the plate was incubated for 30 minutes at room temperature in the dark. Finally, the reaction was stopped with the stop solution, and absorbance was measured at $450 \mathrm{~nm}$ using an automated microplate reader. Recombinant human Dkk-1 was used to generate a linear standard calibration curve (range 31.2$2,000 \mathrm{pg} / \mathrm{ml}$ ). The manufacturer-reported precision was 3.3-4.2\% (intra-assay) and 4.6-7.6\% (inter-assay). The sensitivity of this assay was $4.2 \mathrm{pg} / \mathrm{ml}$.

\section{Statistical analysis}

Statistical analysis was carried out using the statistical package for social sciences (SPSS) software, version 16.0 for Windows. Tests of normality and test of homogeneity 
of variances were performed to determine the subject's age, body mass index (BMI) and Dkk-1 concentration in the plasma and synovial fluid. The analysis of co-variance (ANCOVA) indicated that age, gender and BMI were not potentially confounding factors in the study. Demographic data between patients and controls were compared by Chi-square tests and unpaired Student's $t$ tests, where appropriate. Comparisons between the groups were performed using one-way analysis of variance (ANOVA) with Tukey post hoc test if ANOVA showed significance. Comparisons between groups were made using Mann-Whitney $U$ test (for two groups) or KruskalWallis test (for more than two groups) when the variances were not equal among the groups. Pearson's correlation coefficient was employed to determine the correlation among the concentration of Dkk-1 in the plasma and synovial fluid and the disease severity. Sensitivity, specificity, receiver-operating characteristic (ROC) curves were also determined. $\mathrm{P}$ values $<0.05$ were considered to be statistically significant for differences and correlations. All values are expressed as mean \pm standard deviation (SD) and 95\% confidence intervals (95\%CI).

\section{Results}

Thirty-five plasma and synovial fluid samples from knee OA patients and 15 plasma samples from healthy controls were acquired for measurement of Dkk-1 concentrations. Characteristics of the study population are shown in Table 1 . There was no clinically meaningful difference in age between OA patients and controls $(68.8 \pm 8.2$, 95\%CI $66.3-70.5$ vs $67.5 \pm 4.6$, 95\%CI $65.3-$ 70.3 years, $\mathrm{p}=0.6)$. In addition, the female $/ \mathrm{male}$ ratio was $26 / 9$ in patients and $10 / 5$ in controls $(p=0.1)$. The study population was adjusted for age and gender. There was no significant difference in body mass index between OA patients and controls (26.6 $\pm 3.8,95 \%$ CI $25.3-28.0$ vs $25.5 \pm 1.3,95 \%$ CI $24.6-26.4 \mathrm{~kg} / \mathrm{m}^{2}$, $\mathrm{p}=0.3)$. As demonstrated in Figure 1, OA patients had lower plasma Dkk-1 concentrations compared to healthy controls $(396.0 \pm 258.8,95 \%$ CI 307.1-484.9 vs

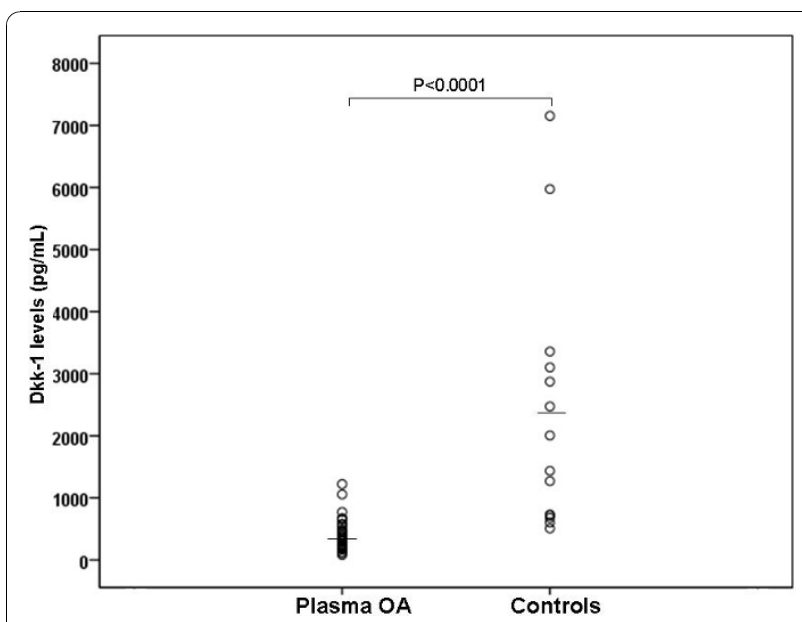

Figure 1 Plasma Dkk-1 levels of patients with osteoarthritis $(n=35)$ and healthy controls $(n=15)$.

$2348.8 \pm 2051.5,95 \%$ CI 1164.3-3533.3 pg/ml, p < 0.0001). Dkk-1 levels in synovial fluid were significantly lower than in paired plasma samples $(58.6 \pm 31.8$, 95\% CI 47.7-69.6 vs $396.0 \pm 258.8,95 \%$ CI $307.1-484.9 \mathrm{pg} / \mathrm{ml}$, $\mathrm{p}<0.001)$. In the OA group, there were no differences in Dkk-1 levels between males and famales in either plasma $(400.7 \pm 275.1,95 \%$ CI $291.9-509.5$ vs $380.1 \pm$ 209.1, 95\%CI 205.3-555.0 pg/ml, p = 0.8) or synovial fluid $(72.1 \pm 60.2$, $95 \%$ CI $21.8-122.5$ vs $54.6 \pm 16.6,95 \%$ CI $48.1-61.2 \mathrm{pg} / \mathrm{ml}, \mathrm{p}=0.5)$. In the control group, plasma Dkk-1 concentrations were not significantly different between both genders (1831.7 \pm 1174.7 , 95\% CI 373.1-3290.3 vs 2636.1 \pm 2426.8 , 95\%CI 770.6$4501.5 \mathrm{pg} / \mathrm{ml}, \mathrm{p}=0.4)$.

According to the Kellgren and Lawrence (KL) classification, 10 patients were KL grade 2, whereas 12 patients were KL grade 3 , and 13 patients were KL grade 4 osteoarthritis. The circulating and synovial fluid levels of Dkk-1 were assessed and compared in association with radiological KL grading of OA. The plasma Dkk-1 levels from KL grade 2 were $711.4 \pm 248.0$, 95\%CI 534.0-888.9

Table 1 Characteristics of knee osteoarthritis patients and controls

\begin{tabular}{|c|c|c|c|c|c|}
\hline & $\begin{array}{l}\text { Controls } \\
(95 \% \mathrm{Cl})\end{array}$ & $\begin{array}{l}\text { OA patients } \\
(95 \% \mathrm{Cl})\end{array}$ & & & \\
\hline & & Total & KL grage 2 & $\mathrm{KL}$ grade 3 & KL grade 4 \\
\hline $\mathrm{N}$ & 15 & 35 & 10 & 12 & 13 \\
\hline \multirow[t]{2}{*}{ Age (years) } & $67.5 \pm 4.6$ & $68.8 \pm 8.2$ & $68.8 \pm 6.8$ & $68.1 \pm 8.3$ & $69.5 \pm 7.5$ \\
\hline & $(65.3-70.3)$ & $(66.3-70.5)$ & (63.9-73.6) & $(61.5-74.6)$ & $(64.9-73.9)$ \\
\hline Female/Male & $10 / 5$ & $26 / 9$ & $7 / 3$ & $9 / 3$ & $10 / 3$ \\
\hline \multirow[t]{2}{*}{ BMI $\left(\mathrm{kg} / \mathrm{m}^{2}\right)$} & $25.5 \pm 1.3$ & $26.6 \pm 3.8$ & $26.2 \pm 1.4$ & $26.4 \pm 3.1$ & $27.2 \pm 4.8$ \\
\hline & $(24.6-26.4)$ & $(25.3-28.0)$ & $(25.2-27.2)$ & $(24.5-28.4)$ & $(23.7-30.7)$ \\
\hline
\end{tabular}

Data are expressed as mean and SD.

$\mathrm{BMI}=$ Body mass index.

$\mathrm{KL}=$ Kellgren and Lawrence. 
$\mathrm{pg} / \mathrm{ml}$; those from KL grade 3 were $340.1 \pm 107.0$, 95\% CI $272.1-408.1 \mathrm{pg} / \mathrm{ml}$; and those from KL grade 4 were $204.9 \pm 86.6,95 \%$ CI 152.6-257.2 pg/ml (Figure 2). These results showed that plasma Dkk-1 levels in KL grade 3 and 4 were significantly lower than those of KL grade 2 ( $\mathrm{p}<0.001)$. Although the mean plasma levels of Dkk-1 in $\mathrm{KL}$ grade 4 were lower than those in KL grade 3, the difference was not significant $(\mathrm{p}=0.1)$. Furthermore, the synovial fluid levels of Dkk-1 from KL grade 2 were $77.3 \pm 51.7,95 \%$ CI $40.3-114.3 \mathrm{pg} / \mathrm{ml}$; those from $\mathrm{KL}$ grade 3 were $58.4 \pm 16.1,95 \% \mathrm{CI} 48.1-68.6 \mathrm{pg} / \mathrm{ml}$; and those from KL grade 4 were $44.5 \pm 10.6$, 95\%CI 38.1 $50.9 \mathrm{pg} / \mathrm{ml}$ (Figure 3). The data showed that synovial fluid Dkk-1 levels in KL grade 2 and 3 were significantly elevated compared with those of KL grade $4(\mathrm{p}=0.04)$. Although synovial fluid Dkk-1 levels in KL grade 2 were elevated compared with those of KL grade 3, the difference was not significant $(\mathrm{p}=0.4)$. We further analyzed the correlation between the plasma and synovial fluid levels of Dkk-1 and the severity of osteoarthritis. Intriguingly, plasma Dkk-1 levels were negatively correlated with the radiographic grading of knee OA $(r=-0.78, p$ $<0.001$ ) (Figure 2). Synovial fluid levels of Dkk-1 were weakly associated with the radiographic severity of disease $(r=-0.42, p=0.01)$ (Figure 3$)$. Figure 4 reveals that a rough estimate of plasma Dkk-1 levels in OA patients is $200-400 \mathrm{pg} / \mathrm{ml}$ whereas that of synovial fluid Dkk-1 levels is 25-70 pg/ml. However, plasma Dkk-1 levels showed a positive correlation with synovial fluid Dkk-1 levels $(\mathrm{r}=0.72, \mathrm{p}<0.001)$. Using ROC curves drawn with data of our results, the cutoff value was set to provide optimal diagnostic accuracy and likelihood ratios for Dkk-1 protein. A plasma level of $459.5 \mathrm{pg} / \mathrm{ml}$

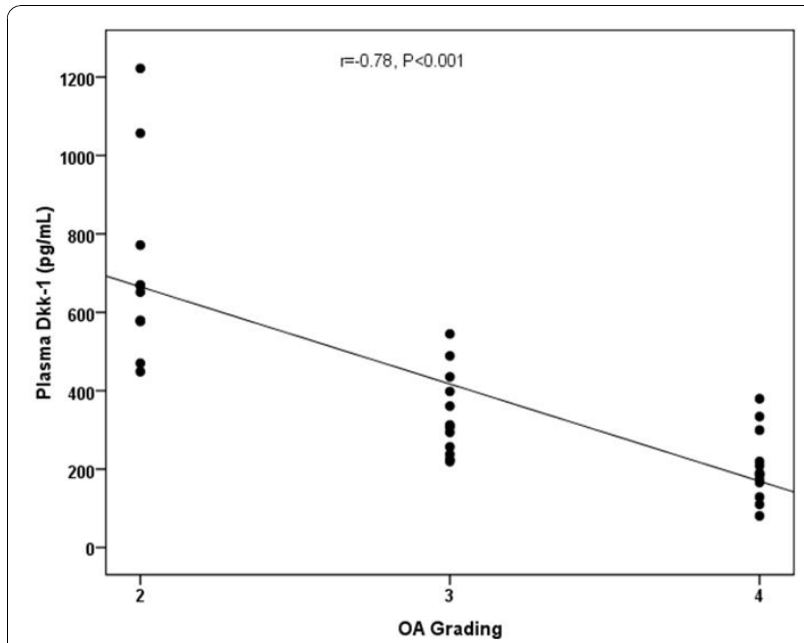

Figure 2 Scattergram showing the inverse correlation between plasma Dkk-1 levels in patients with $O A$ and severity classified

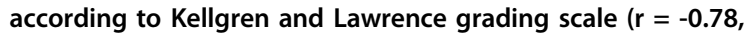
$p<0.001)$

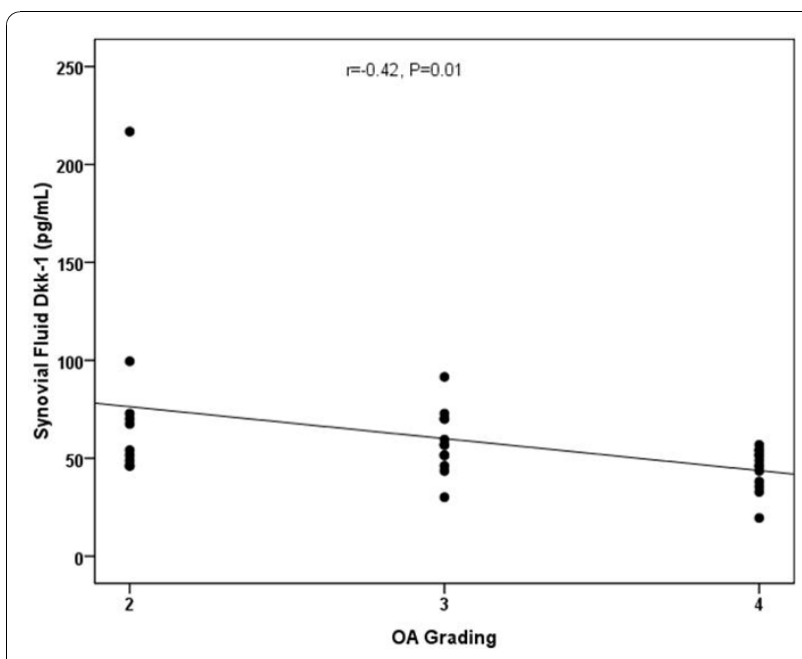

Figure 3 Scattergram showing the negative correlation between synovial fluid Dkk-1 levels in patients with $O A$ and severity classified according to Kellgren and Lawrence grading scale $(r=-0.42, p=0.01)$.

was with a sensitivity of $90 \%$ and a specificity of $92 \%$ for moderate knee OA (KL grade 3). A plasma level of $303.5 \mathrm{pg} / \mathrm{ml}$ was with a sensitivity of $77 \%$ and a specificity of $85 \%$ for advanced knee OA (KL grade 4 ).

\section{Discussion}

The Wnt signaling pathway plays an essential role in cell patterning, proliferation, differentiation, and fate determination during embryogenesis and therefore it is not surprising that Wnt modulators, including Dkks are also involved. Dkk is a family of cysteine-rich proteins consisting of Dkk-1, Dkk-2, Dkk-3, Dkk-4 and a unique

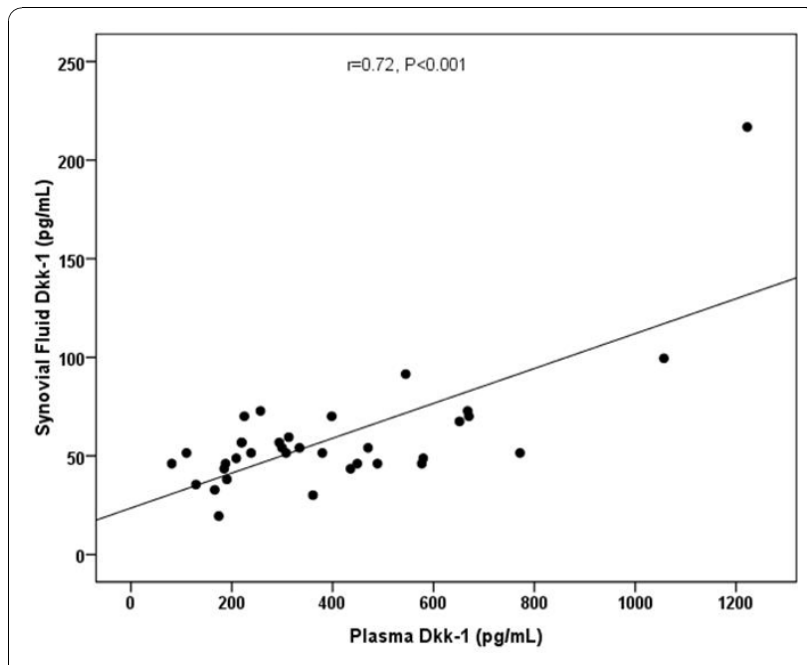

Figure 4 Scattergram showing the positive correlation between plasma and synovial fluid Dkk-1 concentrations in $O A$ patients $(r=0.72, p<0.001)$. 
Dkk-3-related protein "soggy" [19]. Dkk-1 serves as a natural antagonist of the Wnt signaling pathway and plays substantial roles in vertebrate embryogenesis including head induction, skeletal development, and limb patterning [20,21]. Deletion of a single allele of Dkk-1 enhances bone mass in mice [22]. A recent study has demonstrated that aberrant expression of Dkk-1 in myeloma cells was associated with increased bone erosion in human multiple myeloma [23]. Therefore, expression of Dkk-1 in inflammatory and degenerative joint diseases may block bone formation within the joint.

It has been previously demonstrated that circulating Dkk-1 is present in rheumatoid arthritis, ankylosing spondylitis, and osteoarthritis [24-26]. However, the association between circulating and synovial fluid levels of Dkk-1 and disease severity has never been specifically evaluated in knee OA patients. To our knowledge, data on the relationship between Dkk-1 levels in plasma and synovial fluid and severity of knee OA have as yet not been reported in the literature. This study has been the first to illustrate that Dkk-1 was detected in both plasma and synovial fluid derived from patients with primary knee OA, and that Dkk-1 were inversely related to radiographic grading of knee $\mathrm{OA}$.

The most intriguing finding in this study has been that concentrations of Dkk-1 were decreased in plasma of patients with primary knee OA compared to the controls. Our results suggest that there is reduced systemic production of Dkk-1 in knee OA. It should be noted that Dkk-1 levels in synovial fluid were significantly lower than those seen in paired plasma samples. The source of Dkk-1 could be derived from the local tissues (inflamed synovium, cartilage, and subchondral bone) and extraarticular tissues. Previous studies have shown that Dkk-1 was expressed in synovial cells, articular cartilage chondrocytes and subchondral bone osteoblasts in OA knees $[10,27,28]$. Dkk-1 levels in plasma and synovial fluid were measured in a well-defined knee OA population at every stage of disease, and were significantly lower in end-stage knee OA patients compared with early OA patients. This observation suggests a significant reduction in the systemic and local expression of Dkk-1 in patient with advanced knee OA. The mechanisms of Dkk-1 reduction in the circulation and synovial fluid of OA patients remain to be investigated further.

In concordance with our findings, Voorzanger-Rousselot and coworkers have revealed that circulating Dkk-1 levels were lower in patients with knee OA compared with healthy controls [26]. In our study circulating and synovial fluid levels of Dkk-1 were higher in early knee OA patients (KL grade 2) compared to end-stage knee OA (KL grade 4), in agreement with Lane's observation. Recently, Lane and colleagues have documented that increased circulating levels of Dkk-1 appeared to be associated with delayed progression of radiographic hip OA in elderly women [12]. Dkk-1 has been demonstrated to delay new bone formation and subchondral bone remodeling and is a potent negative regulator of osteoblast differentiation [22,23]. Therefore, the mild/moderate knee OA patients with high circulating and synovial fluid levels of Dkk-1 may reflect the Dkk-1 capability to inhibit bone remodeling around the osteoarthritic joint. It is interesting to postulate that Dkk-1 might be able to delay articular cartilage loss. In this study, patients with advanced articular cartilage loss were found to be the subgroup with the lowest circulating and synovial fluid levels of Dkk-1. Previous reports have demonstrated that increased Dkk-1 levels were correlated with the pathogenesis of joint disorders [10,26,27]. In this study, Dkk-1 levels were decreased in direct relation to the severity of knee OA. Moreover, plasma Dkk-1 in OA patients was remarkably lower than in controls. These conflicting results may be attributable to differences in disease advancement, populations or assays applied, or to incomplete control of confounding variables.

It is interesting to point out that in a mouse model of osteoarthritis [27] and in human OA patients [28,29] the loss of a souble Wnt antagonist, frizzled-related protein-3, was associated with enhanced cartilage damage, plausibly by modulating the activity of chondrocytes [30]. This may provide an explanation for the inverse correlation between Dkk-1 and disease severity in primary knee OA patients. Furthermore, decreased Dkk-1 levels tend to be essential for osteophyte formation, suggesting that Wnt signaling is a critical initiator for bone formation in the joint. Local bone formation in the presence of osteophyte formation is a hallmark of degenerative joint diseases including osteoarthritis [10]. Additional studies will be required to determine whether the concentrations of Dkk-1 in synovial fluid and plasma are related to the expression of Dkk-1 in joint tissues. The mechanisms underlying Dkk-1 reduction in the circulation and synovial fluid of OA patients remain to be further investigated.

In this study, we have been aware of several potential limitations. First, our study was based on a small sample size of enrolled patients. A further study conducted on a random sample of a larger population will be needed to substantiate our results. Secondly, only Dkk-1 concentration has been measured in both plasma and synovial fluid. Additional immunohistochemical investigations of Dkk-1 expression could provide further valuable information on the pathogenic role of Dkk-1 in OA. Thirdly, we have not investigated the role of other soluble Wnt inhibitors, such as frizzled-related protein-3, which has been proposed to be associated with joint destruction in OA $[31,32]$. Moreover, the daily variation of plasma Dkk-1 has yet to be fully determined and it is unclear 
which physical activities might influence plasma levels (walking, jumping, eating, etc.). Therefore, diurnal and activity-related variations in plasma Dkk-1 will be needed to be further evaluated. Finally, as this study has been designed as a cross-sectional study it is not possible to determine a definite cause and effect relationship and to arrive at a strong conclusion. However, prospective longitudinal studies are warranted to demonstrate disease progression and define the exact role of Dkk-1 in knee OA.

\section{Conclusions}

This study has revealed a significant decrease in plasma Dkk-1 of OA patients and illustrated a pronounced inverse correlation with the degree of radiographic severity in patients with primary knee OA. The data of this study support the finding that Dkk-1 may be a useful prognostic parameter to reflect the disease severity of primary knee OA. In addition, Dkk-1 levels in plasma were directly correlated with those in synovial fluid. This study has been the first to investigate such a correlation and further studies will be required to define the mechanisms underlying this association. Additional investigations will be required to shed light on the possible role of Dkk-1 involved in the pathogenesis of chronic degenerative joint disorder, with the aim of developing effective pharmacological agents to delay the progression to osteoarthritis.

\footnotetext{
Abbreviations

ANCOVA: analysis of co-variance; ANOVA: analysis of variance; BMl: body mass index; Cl: confidence intervals; DKK-1: Dickkopf-1; ELISA: enzyme-linked immunosorbent assay; KL: Kellgren and Lawrence; LDL: low-density lipoprotein; LRP: low-density lipoprotein receptor-related proteins; OA: osteoarthritis; ROC: receiver-operating characteristic; SD: standard deviation; SPSS: statistical package for social sciences; WNT: Wingless.
}

\section{Acknowledgements}

This research was supported by the Ratchadapiseksompotch Fund, Faculty of Medicine, Chulalongkorn University, the Thailand Research Fund, the Commission on Higher Education, and the National Research Council of Thailand. The authors also would like to thank Prof. Yong Poovorawan and Ms. Petra Hirsch for reviewing the manuscript, Ms. Piyanuch Bumrungpanichthaworn for technical assistance, Mr.Wasan Punyasang for statistical advice, and the Chulalongkorn Medical Research Center (ChulaMRC) for kindly providing facilities.

\section{Author details}

${ }^{1}$ Department of Biochemistry, Faculty of Medicine, Chulalongkorn University, Bangkok 10330, Thailand. 'Department of Orthopaedics, Faculty of Medicine, Chulalongkorn University, Bangkok 10330, Thailand.

\section{Authors' contributions}

$\mathrm{SH}$ was responsible for the conception and design of the study, the analysis and interpretation of the data, and drafting of the manuscript. AT, PY, and SN performed operations, acquired patients, and contributed to the sample collection. NS and ST assisted sample collection and data analysis. All authors have read and approved the final manuscript.

\section{Competing interests}

The authors declare that they have no competing interests.
Received: 1 September 2010 Accepted: 10 November 2010 Published: 10 November 2010

\section{References}

1. Felson DT, Zhang Y, Hannan MT, Naimark A, Weissman BN, Aliabadi P: The incidence and natural history of knee osteoarthritis in the elderly. Arthritis Rheum 1995, 38:1500-1505.

2. Kellgren JH, Lawrence JS: Radiological assessment of osteo-arthrosis. Ann Rheum Dis 1957, 16:494-502.

3. Pelletier JP, Martel-Pelletier J, Abramson SB: Osteoarthritis, an inflammatory disease: potential implication for the selection of new therapeutic targets. Arthritis Rheum 2001, 44:1237-1247.

4. Bejsovec A: Wnt signaling: an embarrassment of receptors. Curr Biol 2000, 10:R919-R922.

5. Zorn AM: Wnt signalling: antagonistic Dickkopfs. Curr Biol 2001, 11: R592-R595.

6. Mao B, Wu W, Li Y, Hoppe D, Stannek P, Glinka A, Niehrs C: LDL-receptorrelated protein 6 is a receptor for Dickkopf proteins. Nature 2001, 411:321-325.

7. Miller JR: The Wnts. Genome Biol 2002, 3:3001.

8. Voorzanger-Rousselot N, Goehrig D, Journe F, Doriath V, Body JJ, Clézardin P, Garnero P: Increased Dickkopf-1 expression in breast cancer bone metastases. Br J Cancer 2007, 97:964-970.

9. Gavriatopoulou M, Dimopoulos MA, Christoulas D, Migkou M, lakovaki M, Gkotzamanidou M, Terpos E: Dickkopf-1: a suitable target for the management of myeloma bone disease. Expert Opin Ther Targets 2009, 13:839-848.

10. Diarra D, Stolina M, Polzer K, Zwerina J, Ominsky MS, Dwyer D, Korb A, Smolen J, Hoffmann M, Scheinecker C, van der Heide D, Landewe R, Lacey D, Richards WG, Schett G: Dickkopf-1 is a master regulator of joint remodeling. Nat Med 2007, 13:156-163.

11. Uderhardt S, Diarra D, Katzenbeisser J, David JP, Zwerina J, Richards W, Kronke G, Schett G: Blockade of Dickkopf (DKK)-1 induces fusion of sacroiliac joints. Ann Rheum Dis 2010, 69:592-597.

12. Lane $N E$, Nevitt MC, Lui LY, de Leon $P$, Corr M: Wnt signaling antagonists are potential prognostic biomarkers for the progression of radiographic hip osteoarthritis in elderly Caucasian women. Arthritis Rheum 2007, 56:3319-3325.

13. Yuasa T, Otani T, Koike T, Iwamoto M, Enomoto-Iwamoto M: Wnt/betacatenin signaling stimulates matrix catabolic genes and activity in articular chondrocytes: its possible role in joint degeneration. Lab Invest 2008, 88:264-274.

14. Honsawek S, Chayanupatkul M, Tanavalee A, Sakdinakiattikoon M, Deepaisarnsakul B, Yuktanandana P, Ngarmukos S: Relationship of plasma and synovial fluid BMP-7 with disease severity in knee osteoarthritis patients: a pilot study. Int Orthop 2009, 33:1171-1175.

15. Honsawek S, Tanavalee A, Sakdinakiattikoon M, Chayanupatkul M, Yuktanandana P: Correlation of plasma and synovial fluid osteopontin with disease severity in knee osteoarthritis. Clin Biochem 2009, 42:808-812.

16. Scanzello CR, Umoh E, Pessler F, Diaz-Torne C, Miles T, Dicarlo E: Local cytokine profiles in knee osteoarthritis: elevated synovial fluid interleukin-15 differentiates early from end-stage disease. Osteoarthritis Cartilage 2009, 17:1040-1048.

17. Pilichou A, Papassotiriou I, Michalakakou K, Fessatou S, Fandridis E, Papachristou G, Terpos E: High levels of synovial fluid osteoprotegerin (OPG) and increased serum ratio of receptor activator of nuclear factorkappaB ligand (RANKL) to OPG correlate with disease severity in patients with primary knee osteoarthritis. Clin Biochem 2008, 41:746-749.

18. Nelson AE, Fang F, Shi XA, Kraus VB, Stabler T, Renner JB: Failure of serum transforming growth factor-beta (TGF-beta1) as a biomarker of radiographic osteoarthritis at the knee and hip: a cross-sectional analysis in the Johnston County Osteoarthritis Project. Osteoarthritis Cartilage 2009, 17:772-776.

19. Niehrs C: Function and biological roles of the Dickkopf family of Wnt modulators. Oncogene 2006, 25:7469-7481.

20. Grotewold $L$, Theil T, Rüther $U$ : Expression pattern of Dkk-1 during mouse limb development. Mech Dev 1999, 89:151-153.

21. Grotewold L, Rüther U: Bmp, Fgf and Wnt signalling in programmed cell death and chondrogenesis during vertebrate limb development: the role of Dickkopf-1. Int J Dev Biol 2002, 46:943-947. 
22. Morvan F, Boulukos K, Clément-Lacroix P, Roman Roman S, Suc-Royer I, Vayssière $B$, Ammann $P$, Martin $P$, Pinho $S$, Pognonec $P$, Mollat $P$, Niehrs $C$ Baron R, Rawadi G: Deletion of a single allele of the Dkk1 gene leads to an increase in bone formation and bone mass. J Bone Miner Res 2006, 21:934-945.

23. Tian E, Zhan F, Walker R, Rasmussen E, Ma Y, Barlogie B, Shaughnessy JD: The role of the Wnt-signaling antagonist DKK1 in the development of osteolytic lesions in multiple myeloma. N Engl J Med 2003, 349:2483-2494.

24. Garnero P, Tabassi NC, Voorzanger-Rousselot N: Circulating dickkopf-1 and radiological progression in patients with early rheumatoid arthritis treated with etanercept. J Rheumatol 2008, 35:2313-2315.

25. Daoussis D, Liossis SN, Solomou EE, Tsanaktsi A, Bounia K, Karampetsou M Yiannopoulos G, Andonopoulos AP: Evidence that Dkk-1 is dysfunctional in ankylosing spondylitis. Arthritis Rheum 2009, 62:150-158.

26. Voorzanger-Rousselot N, Ben-Tabassi NC, Garnero P: Opposite relationships between circulating Dkk-1 and cartilage breakdown in patients with rheumatoid arthritis and knee osteoarthritis. Ann Rheum Dis 2009, 68:1513-1514

27. Weng LH, Wang CJ, Ko JY, Sun YC, Su YS, Wang FS: Inflammation induction of Dickkopf-1 mediates chondrocyte apoptosis in osteoarthritic joint. Osteoarthritis Cartilage 2009, 17:933-943.

28. Weng LH, Wang CJ, Ko JY, Sun YC, Wang FS: Control of Dkk-1 ameliorates chondrocyte apoptosis, cartilage destruction, and subchondral bone deterioration in osteoarthritic knees. Arthritis Rheum 2010, 62:1393-1402.

29. Lories RJ, Peeters J, Bakker A, Tylzanowski P, Derese I, Schrooten J, Thomas JT, Luyten FP: Articular cartilage and biomechanical properties of the long bones in Frzb-knockout mice. Arthritis Rheum 2007, 56:4095-4103.

30. Dell'Accio F, De Bari C, El Tawil NM, Barone F, Mitsiadis TA, O'Dowd J, Pitzalis C: Activation of WNT and BMP signaling in adult human articular cartilage following mechanical injury. Arthritis Res Ther 2006, 8:R139.

31. Loughlin J, Dowling B, Chapman K, Marcelline L, Mustafa Z, Southam L: Functional variants within the secreted frizzled-related protein 3 gene are associated with hip osteoarthritis in females. Proc Natl Acad Sci USA 2004, 101:9757-9762.

32. Ikegawa S: New gene associations in osteoarthritis: what do they provide, and where are we going? Curr Opin Rheumatol 2007, 19:429-434.

\section{Pre-publication history}

The pre-publication history for this paper can be accessed here: http://www.biomedcentral.com/1471-2474/11/257/prepub

doi:10.1186/1471-2474-11-257

Cite this article as: Honsawek et al:: Dickkopf-1 (Dkk-1) in plasma and synovial fluid is inversely correlated with radiographic severity of knee osteoarthritis patients. BMC Musculoskeletal Disorders 2010 11:257.

\section{Submit your next manuscript to BioMed Central and take full advantage of:}

- Convenient online submission

- Thorough peer review

- No space constraints or color figure charges

- Immediate publication on acceptance

- Inclusion in PubMed, CAS, Scopus and Google Scholar

- Research which is freely available for redistribution

Submit your manuscript at www.biomedcentral.com/submit
Biomed Central 